\title{
Investigation of the Postoperative Complications Rate and Predictors in Patients Undergoing Surgery due to Associated Carotid and Coronary Occlusive Disease
}

\author{
Vladimir Jovicic, MD, ${ }^{1}$ Svetozar Putnik, MD, PhD,${ }^{1}$ Aleksandar Djordjević, MD,${ }^{1}$ Anita Grgurević, MD, PhD, ${ }^{2}$ \\ Igor Atanasijević, MD, ${ }^{3}$ Dusko Terzic, $\mathrm{MD},{ }^{1}$ Milica Jovicic, $\mathrm{MD}, \mathrm{PhD}^{4}$ \\ ${ }^{1}$ Clinic for Cardiac Surgery, Clinical Centre of Serbia, Faculty of Medicine, University of Belgrade, Belgrade, Serbia; ${ }^{2}$ Institute of \\ Epidemiology, Faculty of Medicine, University of Belgrade, Belgrade, Serbia; ${ }^{3}$ Institute of Cardiovascular Diseases "Dedinje", Belgrade, \\ Serbia; ${ }^{4}$ Institute of Rehabilitation, Faculty of Medicine, University of Belgrade, Belgrade, Serbia
}

\section{ABSTRACT}

Background: The aim of this study was to evaluate the frequency of postoperative complications in patients who underwent coronary artery bypass grafting (CABG) and simultaneous carotid endarterectomy (CEA) and find predictors of postoperative complications.

Methods: We retrospectively evaluated 86 patients after simultaneous CABG and CEA. Inclusion criteria were: patients with asymptomatic carotid stenosis with a reduction of the carotid lumen diameter of more than $70 \%$ detected with Doppler ultrasound and diagnosed with one, two, or three vessel coronary artery disease with coronary stenosis more than $75 \%$ and hemodynamic significant stenosis of the left main artery. Exclusion criteria were patients with urgent and previous cardiac surgery and patients with myocardial infarction and stroke in the past one month. We monitored preoperative (ejection fraction, coronarography status), operative (number of grafts, on-pump or off-pump technique) and postoperative (extubation, unit care and hospital stay, bleeding and reoperation) details and complications (myocardial infarction, neurological events, inotropic agents and transfusion requiry, infection, arrhythmic complication, renal failure, mortality).

Results: Postoperative complications were observed in 18 (29.9\%) patients. Two patients (2.3\%) had postoperative stroke and one patient (1.2\%) had transient ischemic attack (TIA). Previous stroke was a predictor for increased postoperative neurological events $(P<.05)$. Intrahospital mortality was $8.1 \%$.

Conclusion: Simultaneous CEA and CABG were performed with low rates of stroke and TIA. Previous stroke was identified as a predictor for increased postoperative neurological complications.

\section{INTRODUCTION}

Concomitant carotid and coronary disease is a common problem and an important risk factor of coronary artery

Received March 19, 2018; received in revised form November 8, 2018; accepted November 14, 2018.

Correspondence: Milica fovicic, Institute of Rebabilitation, Sokobanjska 17, Belgrade;0641180725; (e-mail: medi@eunet.rs). bypass grafting (CABG)-associated stroke. The risk of cerebrovascular accident has been reported to increase up to $14 \%$ in patients with severe carotid artery stenosis [Huh 2003; Khaitan 2000]. There is no consensus as to the optimal management of patients with concomitant carotid and coronary disease [Naylor 2010]. Also, treatment strategies are not supported by studies with level 1 evidence [Cywinski 2006]. One of the strategies includes simultaneous CABG and CEA that is associated with low rates of mortality and morbidity [Eren 2005; Levy 2012; Ren 2012; Yuan 2009]. On the other hand, a systematic review of 97 studies in patients treated with CEA and CABG showed that the highest rate of stroke and death was noticed in the group of patients where these procedures were performed simultaneously [Naylor 2003]. There is insufficient data in domestic literature regarding the outcome of simultaneous operative treatment of patients with associated carotid and coronary occlusive disease. Thus, the purpose of this study was to evaluate postoperative complications of patients who underwent CABG and simultaneous CEA, with particular reference to neurological events. Also we wanted to identify significant predictors that were associated with postoperative outcome.

\section{METHODS}

The retrospective cohort study with a short-term followup period was conducted in order to identify predictors of postoperative complications. The study included 86 patients who underwent simultaneous CABG and CEA during the same anesthetic setting in a 4-year period from 2012 to 2016 (Clinic for Cardiac Surgery, Clinical Centre of Serbia, Medical School, University of Belgrade). The observed follow-up period was duration of hospitalization after surgical treatment. The study was approved by the Medical School, University of Belgrade research ethics committee. The indications for simultaneous procedure were: patients with asymptomatic carotid stenosis with a reduction of the carotid lumen diameter of more than $70 \%$ detected with Doppler ultrasound and diagnosed with one, two, or three vessel coronary artery disease with coronary stenosis more than $75 \%$ and hemodynamic significant stenosis of the left main artery. Exclusion criteria were patients with urgent and previous cardiac surgery and patients with myocardial infarction and stroke in the 
Table 1. Demographic, Preoperative, and Operative Characteristics

\begin{tabular}{lc}
\hline & $\mathrm{n}=86$ \\
\hline Age, mean \pm SD & $67.2 \pm 7.2$ \\
Sex, $\mathrm{n}(\%)$ & \\
$\quad$ Male & $57(66.3)$ \\
Female & $29(33.7)$ \\
Comorbidity, $\mathrm{n}(\%)$ & \\
Hypertension & $83(96.5)$ \\
Hyperlipidemia & $63(73.2)$ \\
Diabetes mellitus & $34(39.5)$ \\
COPD & $10(11.9)$ \\
Renal failure & $1(1.2)$ \\
TIA & $1(1.2)$ \\
Stroke & $5(6)$ \\
EF (\%), mean \pm SD & $51.2 \pm 11.9$ \\
Coronary disease, $\mathrm{n}(\%)$ & \\
One-vessel & $3(3.5)$ \\
Two-vessel & $10(11.6)$ \\
Three-vessel & $42(48.8)$ \\
Left main artery & $31(36)$ \\
Off-pump CABG, $\mathrm{n}(\%)$ & $12(14)$ \\
On-pump CABG, $\mathrm{n}(\%)$ & $74(86)$ \\
Number of grafts, med (IR) & $3(1)$ \\
\hline
\end{tabular}

COPD indicates chronic obstructive pulmonary disease; TIA, transient ischemic attack; $E F$, ejection fraction; med, median; IR, interquartile range.

past one month. Our strategy was that after the CAE (done by the vascular team), the wound was completely closed on the neck, and then the off-pump CABG procedure was done. If an extracorporeal circulation (ECC) was necessary for a given patient, it was advantageous for the bypass on a full to create proximal anastomosis. Regardless of the choice of CABG procedure, full heparinization was applied (400 IU/kg). Patients monitoring during hospitalization included demographic characteristics (age and sex), comorbidity, preoperative (ejection fraction $[\mathrm{EF}]$, coronarography status), operative (number of grafts, on-pump or off-pump technique) and postoperative (extubation, unit care stay, hospital stay, bleeding, and reoperation) details, complications (myocardial infarction [MI], neurological events, inotropic agents and transfusion requiry, infection, arrhythmic complication, renal failure, mortality). The multidisciplinary team participated in the treatment of the patients. On suspicion of a neurological event, a neurologist was consulted who, after a neurological examination, indicated CT scan of the endocranium based on which the diagnosis of stroke was made. Statistical analyses were performed using IBM SPSS version 24.0 packaged software and included
Table 2. Postoperative Characteristics and Complications

\begin{tabular}{lc}
\hline & $\mathrm{n}=86$ \\
\hline Extubation, days, median (IR) & $1(1)$ \\
Intensive unit stay, days, med (IR) & $4(2)$ \\
Hospital stay, days, mean \pm SD & $11 \pm 6.2$ \\
Transfusion, $\mathrm{n}(\%)$ & $48(55.8)$ \\
Complications, $\mathrm{n}(\%)$ & $18(20.9)$ \\
Inotropic support, $\mathrm{n}(\%)$ & $20(23.2)$ \\
Reoperation for bleeding, $\mathrm{n}(\%)$ & $5(5.8)$ \\
Hemodialysis, $\mathrm{n}$ (\%) & $6(7)$ \\
Stroke, $\mathrm{n}(\%)$ & $2(2.3)$ \\
TIA, $\mathrm{n}(\%)$ & $1(1.2)$ \\
Mediastinitis, $\mathrm{n}(\%)$ & $1(1.2)$ \\
Arrhythmic complications, $\mathrm{n}(\%)$ & $7(8.1)$ \\
Mortality, $\mathrm{n}(\%)$ & $7(8.1)$ \\
\hline
\end{tabular}

IR indicates interquartile range; TIA, transient ischemic attack.

methods of descriptive statistics (measures of central tendency [mean and median], measures of variability [standard deviation], interquartile range [IQR], relative numbers and methods of analytic statistics [chi square test, Student t test, Pearson and Spearman correlations). Cox regression analysis was used to identify predictors of postoperative complications.

\section{RESULTS}

Demographic, preoperative, and operative data are summarized in Table 1. The mean age of patients was $67.2 \pm$ 7.2 years. Of the 86 patients, $57(66.3 \%)$ were men, and 29 $(33.7 \%)$ were women. A history of hypertension was present in 83 patients (96.5\%). Hyperlipidemia and diabetes mellitus (DM) were present in $63(73.2 \%)$ and $34(39.5 \%)$ patients, respectively. Five patients (6\%) had a history of previous stroke and one patient $(1.2 \%)$ had a history of previous transient ischaemic attack (TIA). Ten patients (11.6\%) reoprted chronic obstructive pulmonary disease (COPD) and one patient $(1.2 \%)$ reported renal failure (Table 1$)$. The mean ejection fraction (EF) was $51.2 \pm 11.9$ (range: $21 \%$ to $75 \%$ ). A large number of patients (48.8\%) had three vessel coronary disease, $36 \%$ of patients had left main disease. $3.5 \%$ and $11.6 \%$ of patients had one and two vessel coronary disease, respectively (Table 1$)$. The majority of patients $(86 \%)$ underwent on-pump CABG and the median number of grafts was 3 (Table 1). Postoperative characteristics and complications are listed in Table 2. Eighteen patients (20.9\%) developed complications including 2 strokes (2.3\%), 1 TIA (1.2\%), 7 arrythmic complications (8.1\%), 6 renal failure requiring hemodylisis $(7 \%), 5$ reoperations for bleeding $(5.8 \%), 1$ mediastinitis $(1.2 \%)$. Twenty patients $(23.2 \%)$ required the consumption of 
Table 3. Variables Associated with Postoperative Stroke and Mortality

\begin{tabular}{lcccc}
\hline \multicolumn{2}{c}{ Postoperative stroke } & & Mortality \\
Variables & $\mathrm{RR}(\mathrm{Cl})$ & $P$ & $\mathrm{RR}(\mathrm{Cl})$ & $P$ \\
\hline $\begin{array}{l}\text { Previous stroke } \\
\text { Postoperative complications }\end{array}$ & $15.8(1.02-252.6)$ & .049 & $2.63(0.32-21.88)$ & .370 \\
\hline
\end{tabular}

$\mathrm{RR}$ indicates relative risk; $\mathrm{Cl}$, confidence interval.

inotropic agents and 48 patients $(55.8 \%)$ required transfusion. The average length of unit care and hospital stay was 4 and 11 \pm 6.2 days, respectively (Table 2). In-hospital deaths occured in 7 patients $(8.1 \%)$ (Table 2). Of the 7 patients, 4 were men and 3 were women. Causes of deaths were neurologic events (strokes) in two patients, arrhythmic complications in one patient (atrial fibrilation in the early postoperative course), heart failure in one patient. The cause of death of three patients were not neurological and cardiac complications

Regression analysis revealed some predictors for mortality and postoperative strokes (Table 3). We found significant correlation between postoperative complications and mortality rate. Patients with complications had higher mortality rate $(\rho$ $=-0.370 ; P<.001)$ and presence of complications was significantly associated with in-hospital mortality $(P=.007)$ (Table $3)$. Hospital stay correlated weakly with mortality $(\rho=-0.256$; $P=.041$ ). Patients with longer hospital stay had higher mortality rate but hospital stay was not significantly associated with mortality rate $(P=.088)$. Patients with three or more associated diseases had a higher incidence of mortality compared to patients with fewer associated illnesses but without significant difference $(P=.095)$. Individually, previous stroke was not significantly associated with mortality rate $(P>.05)$ (Table 3$)$. In the group of patients with previous stroke, we found higher prevalence of postoperative neurologic events (20\%) in relation to group of patients without previous stroke $(1.3 \%)$ and that was statistically significant $\left(\chi^{2}=7.101 ; P=.008\right)$. Previous stroke was significantly associated with the prevalence of postoperative neurologic events $(P<.05)$ (Table 3$)$.

\section{DISCUSSION}

Concomittant carotid and coronary disease is a very frequent clinical problem. In the associated coronary and carotid disease, a multidisciplinary team composed of cardiac surgeons, cardiologists, anesthesiologists, neurologists, transfusiologists, and vascular surgeons has established the protocol of the Clinical Center of Serbia according to which the primary coronary operation without previous carotid operation is carried out in the following conditions: Asymptomatic unilateral stenosis of the carotid arteries less than $80 \%$ or previous stroke with a carotid stenosis of less than $80 \%$. Primary carotid operation without previous coronary operation-carotid stent is performed in patients with acute neurological symptoms with bilateral lesions of carotid arteries greater than $50 \%$ with or without contralateral occlusion or in the case of a stroke linked to carotid stenosis greater than $80 \%$ in the case of stable chronic angina in the absence of a recent infarction. Simultaneous surgery in patients with both carotid and coronary disease is performed in patients with unstable angina pectoris, left main stenosis, and diffuse coronary disease without satisfactory collaterals. The basic controversy is about a combined procedure in patients with asymptomatic carotid artery stenosis greater than $80 \%$, and in this case the team decision adjusts to individual patients characteristics. Postoperative stroke is one of the most concerning complications after CABG, with reported $24.8 \%$ mortality [John 2000]. A $9.2 \%$ rate of stroke or TIA in cardiac surgical patients with asymptomatic carotid stenosis is reported when compared with $1.3 \%$ of patients with no carotid stenosis [Brener1987]. The possible mechanism is via embolization from unstable plaques or by decreased cerebral blood flow distal to critical stenosis. Considering the patient's risk profile, lesion morphology and symptom status, simultaneous surgical strategy (CEA before CABG) may be considered. The main goal is stroke prevention or reduction of the stroke rate. Thus, the purpose of this study was to provide a description of simultaneous CEA and CABG outcome and find possible factors that were associated with patient's postoperative status. The majority of studies reported postoperative complications in relation to stroke and mortality [Aydin 2014; Nwakanma 2006; Gansera 2012; Illuminati 2011]. We primarily examined the frequency of stroke and mortality, but we also investigated and described other complications that might be expected during the postoperative course. The first studies on combined surgical approach showed a reduced incidence of postoperative complications primarily in the sense of stroke and myocardial infarction in simultaneous CEA and CABG [Trachiotis 1997]. On the other hand, there are studies that report a significantly higher percentage of postoperative complications (stroke $4.48 \%$, TIA $1.66 \%$, and intrahospital mortality $3.56 \%$ ) relative to reference groups [Gopaldas 2011; Prasad 2010]. The authors of these studies emphasized the importance of further detailed analysis and scientific research when it comes to the choice of treatment of patients with associated carotid occlusive and coronary disease. Generally speaking, the majority of patients in our study did not have complications during the early postoperative period $(79.1 \%)$ and $20.9 \%$ of patients had complications. The most frequent complication was inotropic support $(31.3 \%)$, while the least frequent complications were TIA (1.2\%) and mediastinitis (1.2\%). Similarly, the rate 
of TIA (1.2\%) was close to the results by Aydin et al $(0.9 \%)$ [Aydin 2014]. A higher percent of patients with postoperative TIA (2\%) was observed in the study by Kolh et al [Kolh 2006]. There were no patients with myocardial infarction in the early postoperative period and that is in accordance wtih other studies [Aydin 2014; Nwakanma 2006]. Arrhythmic complications in our study were higher $(5.8 \%)$ in relation to the results by Aydin et al (1\%) [Aydin 2014]. The highest rate of arrhythmic complication (18.5\%) was reported in the study by Nwakanma et al [Nwakanma 2006]. 5.9\% of patients were reoperated due to postoperative bleeding, $7 \%$ of patients required hemodialysis. In other studies, a number of reoperations were observed (8.04\%) [Prasad 2010] and there were no patients with acute renal insufficiency [Nwakanma 2006]. Simultaneous CEA and CABG in our study population were performed with low rates of stroke $(2.3 \%)$ and TIA $(1.2 \%)$ and that is in accordance with other studies [Aydin 2014]. The authors of a previous study reported the postoperative stroke rate of $2 \%$ and postoperative TIA rate of $0.9 \%$ [Aydin 2014]. Similar results were observed in other research papers [Levy 2012; Kougias 2007; Naylor 2011]. In the study by Levy et al [Levy 2012], the prevalence of postoperative stroke was $2.5 \%$ while the study by Naylor et el [Naylor 2011] and Kougias et al [Kougias 2007] reported the prevalence of postoperative stroke at $2.7 \%$. A higher frequency of postoperative strokes (3.7\%) was registered by Nwakanma et al [Nwakanma 2006], but the mean age of the patients in their study was higher in relation to our study and they reported a higher frequency of emergency surgery (40.7\%). In our study, all patients underwent elective surgery. Also, a higher percent of postoperative neurological events (stroke: $3.9 \%$ ) was noticed in the study by Timaran et al [Timaran 2008]. In our study, both patients with postoperative stroke were from the "on-pump" group, which was expected considering systemic hypotension, and in combination with stenosis of intracranial arteries, it can lead to brain hypoperfusion. It has been shown that this phenomenon is responsible for $8.8 \%$ of strokes in patients undergoing CABG [Likosky 2003]. In our study, seven patients died $(8.1 \%)$, neurological and cardiac complications were not the cause of the death of three patients, one patient died due to atrial fibrillation in the early postoperative course, one patient had heart failure, while the cause of death of two patients was related to neurological complications (stroke). There was a strong correlation between postoperative complications and mortality rate. Also, in the group of patients with complications, a higher incidence of mortality was observed, which was statistically significant. Similar results were observed by Kolh et al [Kolh 2006]. In our study, the regression analysis revealed that the presence of postoperative complications was the predictor of mortality $(P=.007)$ (Table 3$)$. Patients with three or more associated diseases had a higher incidence of mortality compared to patients with fewer associated illnesses, and this correlation was close to the statistical significance level, while in the regression model it did not distinguish itself as a significant predictor. This is comparable with results by Busch et al [Busch 1999]. The results of regression analyses in literature data indicate that socio-demographic characteristics (age), comorbidity (cardiac insufficiency), and postoperative characteristics (reintubation) are predictors of mortality [Nwakanma 2006; Gansera 2012]. In our study, comorbidity (previous stroke) was a predictor of postoperative stroke $(P<.05)$. The results of this study showed that simultaneous CEA and CABG were performed with low rates of stroke and TIA. On the other hand, the rate of mortality was high $(8.1 \%)$. These results should have a direct clinical application and influence on the optimal decision-making and plan in terms of the details of surgical treatment of coronary disease in patients with simultaneous and associated carotid occlusive disease. The limiting factors of this study are the lack of a control group and evaluation of long-term results.

\section{Conclusion}

Simultaneous CEA and CABG were performed with low rates of stroke and TIA and previous stroke was identified as a predictor for increased postoperative neurological events. Further studies are needed to maintain the long-term follow-up.

\section{REFERENCES}

Aydin E, Ozen Y, Sarikaya S, Yukseltan I. 2014. Simultaneous coronary artery bypass grafting and carotid endarterectomy can be performed with low mortality rates Cardiovasc J Afr 25:130-3.

Brener BJ, Brief DK, Alpert J, Goldenkranz RJ, Personnet V. 1987. The risk of stroke in patients with asymptomatic carotid stenosis undergoing cardiac surgery: a follow-up study. J Vasc Surg 5:269-79.

Busch T, Sirbu H, Aleksic I, et al. 1999. Combined approach for internal carotid artery stenosis and cardiovascular disease in septuagenarians-a comparative study. Eur J Cardiothorac Surg 16:602-6.

Cywinski JB, Koch CG, Krajewski LP, Smedira N, Li L, Starr NJ. 2006. Increased risk associated with combined carotid endarterectomy (CEA) and coronary artery bypass graft surgery: a propensity-matched comparison with isolated coronary artery bypass graft surgery. J Cardiothorac Vasc Anesth 20:796-802.

Eren E, Balkanay M, Toker ME, et al. 2005. Simultaneous carotid endarterectomy and coronary revascularization is safe using either on-pump or off-pump technique. Int Heart J 46:783-93.

Gansera B, Schmidtler F, Weingartner J, et al. 2012. Simultaneous carotid endarterectomy and cardiac surgery: early results of 386 patients. Thorac Cardiovasc Surg 60:508-16.

Gopaldas RR, Chu D, Dao TK, et al. 2011. Staged versus synchronous carotid endarterectomy and coronary artery bypass grafting: analysis of 10-year nationwide outcomes. Ann Thorac Surg 91:1323-9.

Huh J, Wall M, Soltero E. 2003. Treatment of combined coronary and carotid artery disease. Curr Opin Cardiol 18:447-53.

Illuminati G, Ricco J-B, Caliò F, et al. 2011. Short-term results of a randomized trial examining timing of carotid endarterectomy in patients with severe asymptomatic unilateral carotid stenosis undergoing coronary artery bypass grafting. J Vasc Surg 54:993-9.

John R, Choudhri AF, Weinberg AD, et al. 2000. Multicenter review of preoperative risk factors for stroke after coronary artery bypass grafting. Ann Thorac Surg 69:30-5.

Khaitan L, Sutter FP, Goldman SM, et al. 2000. Simultaneous carotid endarterectomy and coronary revascularization. Ann Thorac Surg 69:421-4. 
Kolh PH, Comte L, Tchana-Sato V, et al. 2006. Concurrent coronary and carotid artery surgery: factors influencing perioperative outcome and long-term results. Eur Heart J 27:49-56.

Kougias P, Kappa JR, Sewell DH, Feit RA, Michalik RE. 2007. Simultaneous carotidendarterectomy and coronary artery bypass grafting: results in specific patient groups. Ann Vasc Surg 21:408-14.

Levy E, Yakubovitch D, Rudis E, et al. 2012. The role of combined carotid endarterectomy and coronary artery bypass grafting in the era of carotid stenting in view of long-term results. Interact Cardiovasc Thorac Surg 15:984-8.

Likosky DS, Marrin CA, Caplan LR, et al. 2003. Determination of etiologic mechanisms of strokes secondary to coronary artery bypass graft sur $\neg$ gery. Northern New England Cardiovascular Disease Study Group Stroke 34:2830-4.

Naylor AR, Cuffe RL, Rothwell PM, Bell PR. 2003. A systematic review of out-comes following staged and synchronous carotid endarterectomy and coronary artery bypass. Eur J Vasc Endovasc Surg 25:380-9.

Naylor AR. 2010. Managing patients with symptomatic coronary and carotid artery disease. Perspect Vasc Surg Endovasc Ther 22:70-6.

Naylor AR, Bown MJ. 2011. Stroke after cardiac surgery and its association with asymptomatic carotid disease: an updated systematic review and metaanalysis. Eur J Vasc Endovasc Surg 41:607-24.

Nwakanma L, Poonyagariyagorn HK, Bello R, Khoynezhad A, Smego
D, Plestis KA. 2006. Early and late results of combined carotid endarterectomy and coronary artery bypass versus isolated coronary artery bypass. Interact Cardiovasc Thorac Surg 5:159-65.

Prasad SM, Shuang Li, Rankin JS, et al. 2010. Current outcomes of simultaneous carotid endarterectomy and coronary artery bypass graft surgery in North America. World J Surg 34:2292-98.

Ren S, Liu P, Ma G, Wang F, Qian S, Fan X. 2012. Long-term outcomes of synchronous carotid endarterectomy and coronary artery bypass grafting versus solely carotid endarterectomy. Ann Thorac Cardiovasc Surg $18: 228-35$

Timaran CH, Rosero EB, Smith ST et al. 2008. Trends and outcomes of con $\neg$ current carotid revascularization and coronary bypass. J Vasc Surg 48:355-60.

Trachiotis GD, Pfister AJ. 1997. Management strategy for simultaneous carotid endarterectomy and coronary revascularization. Ann Thorac Surg 64:1013-18. (Akins LW, Moncure AC, Daggett WM. 1995. Safety and efficiency of concomitant carotid and coronary artery operations Ann Thorac Surg 60:311-17.)

Yuan SM, Wu HW, Jing H. 2009. Treatment strategy for combined carotid artery stenosis and coronary artery disease: staged or simultaneous surgical procedure? Tohoku J Exp Med 219:243-50. 